\title{
BRUCELOSE BOVINA NO ESTADO DE RORAIMA: ESTUDO RETROSPECTIVO
}

\section{L.P.A. Souza, A.W.L. Brasil, R.N. Parentoni, E.O. Azevedo, C.J. Alves, S.S. Azevedo}

Universidade Federal de Campina Grande, Centro de Saúde e Tecnologia Rural, Unidade Acadêmica de Medicina Veterinária, Av. Universitária s/no, CEP 58700-970, Patos, PB, Brasil. E-mail: ssazevedo@cstr. ufcg.edu.br

\section{RESUMO}

O objetivo do presente trabalho foi determinar a frequência de propriedades positivas (focos) e de fêmeas bovinas soropositivas para a brucelose no Estado de Roraima, bem como identificar fatores de risco. Foram utilizados dados da Agência de Defesa Agropecuária do Estado de Roraima (ADERR), coletados de suas quatro microrregiões, durante o período de janeiro de 2007 a julho de 2009. Durante esse período, foram examinadas 387 propriedades, e 9.087 soros de fêmeas bovinas com idade $\geq 24$ meses foram submetidos ao diagnóstico sorológico da brucelose. Para a análise de fatores de risco, foi utilizada a regressão logística múltipla. Uma propriedade foi considerada foco quando apresentou pelo menos um animal soropositivo. Das propriedades investigadas, 106 $(27,4 \%)$ apresentaram pelo menos um animal soropositivo e, dos animais analisados, $369(4,1 \%)$ foram soropositivos. Os fatores de risco identificados foram: possuir mais de 51 animais no rebanho (odds ratio $=1,87 ; \mathrm{p}=0,015$ ) e alugar pasto (odds ratio $=2,20 ; \mathrm{p}=0,003$ ). Sugere-se que esforços sejam concentrados na intensificação da vacinação de bezerras no Estado de Roraima com o intuito de que seja alcançada uma prevalência compatível com as ações de teste e sacrifício de animais.

PALAVRAS-CHAVE: Brucellaabortus, bovino, frequência, fatores de risco, epidemiologia (controle).

\section{ABSTRACT}

BOVINE BRUCELLOSIS IN RORAIMA STATE: RETROSPECTIVE STUDY. The aim of this study was to determine the frequency of positive herds (foci) and seropositive bovine females for brucellosis in the state of Roraima, Northern region of Brazil, as well as to identify risk factors. Data from the Agency of Agricultural Protection in the state, collected from its four microregions during the January 2007 to July 2009 period, were used. During this period, 387 herds were examined, and 9,087 adult bovine female sera were submitted to the serological diagnosis of brucellosis. For risk factor analysis the multiple logistic regression was used. A herd was considered focus when presented at least one seropositive animal. Of the herds investigated, $106(27.4 \%)$ had at least one seropositive animal, and of the animals examined, $369(4.1 \%)$ were seropositive. Risk factors identified were: herd size larger than 51 animals (odds ratio $=1.87 ; \mathrm{p}=0.015$ ) and pasture rental (odds ratio $=2.20 ; p=0.003$ ). It is suggested that efforts must be concentrated on the intensification of heifer vaccination in the State of Roraima in order to reach a prevalence value compatible with actions of test and sacrifice of animals.

KEY WORDS: Brucella abortus, bovine, frequency, risk factors, epidemiology (control).

\section{INTRODUÇÃO}

A brucelose bovina é uma doença bacteriana de evolução crônica e caráter granulomatoso difuso, caracterizada pela infecção de células do sistema mononuclear fagocitário, causada por uma bactéria intracelular facultativa integrante do gênero Brucella, e apresentando-se em todo o mundo como problema sanitário e econômico (PAULIN; FERREIRA Neto, 2003). O principal agente etiológicoéa Brucella abortus, cujo biotipo 1 é o mais frequente (АсHA; SZYFRES, 2001).
Na maioria dos países, a brucelose bovina ainda é um sério problema de saúde animal (MEGID et al., 2000). Com exceção daqueles que, após programas rigorosos, conseguiram erradicá-la ou pelo menos reduzir significativamente a prevalência, nos países em desenvolvimento ainda se trata de uma questão sanitária preocupante (MATHIAS et al., 2007). No Brasil, a brucelosebovina aindaéendêmica, com prevalências mais elevadas em regiões com maior densidade de bovinos (Poester et al., 2002; PAulin; Ferreira Neto, 2003).

Com a instituição do Programa Nacional de Controle e Erradicação da Brucelose e Tuberculose 
Animal (PNCEBT) pelo Ministério da Agricultura, Pecuária e Abastecimento (MAPA), em 2001 (BRASIL, 2001), foram planejados inquéritos sorológicos como objetivo de determinar a situação epidemiológica da brucelose bovina nas Unidades Federativas edirecionar a escolha das estratégias de controle adequadas, que podem diferir de acordo com a frequência e a distribuição da doença. Até o momento, os inquéritos foram conduzidos em 15 Estados, e as prevalências de animais soropositivos variaram de 0,06\%, em Santa Catarina, a 10,2\%, em Mato Grosso (Alves et al., 2009; Azevedo et al., 2009; CHATE et al., 2009; DiAs et al., 2009a; DiAs et al., 2009b; GONÇALVES et al., 2009a; GONÇALVES et al., 2009b; KLEIN-GUNNEWIEK et al., 2009; MARVulo et al., 2009; NEgreiros et al., 2009; OGATA et al., 2009; Rocha et al., 2009; SIKUSAWA et al., 2009; SILVA et al., 2009; Villar et al., 2009). Contudo, no Estado de Roraima o inquérito ainda não foi iniciado.

Dessa maneira, o presente trabalho foi estruturado com o objetivo de determinar a frequência de propriedades positivas e de animais soropositivos para a brucelose bovina no Estado de Roraima, utilizando dados do Serviço de Defesa Agropecuária do Estado relativos aos relatórios emitidos por médicos veterinários habilitados pelo MAPA para o sorodiagnóstico da brucelose bovina, no período de janeiro de 2007 a julho de 2009.

\section{MATERIAL E MÉTODOS}

\section{Caracterização do Estado}

O Estado de Roraima possui uma população de 451.227 habitantes, distribuída em 15 municípios e quatro microrregiões geográficas, a saber: Boa Vista (municípios de Alto Alegre, Amajari, Pacaraima e Boa Vista), Caracaraí (municípios de Iracema, Caracaraí e Mucajaí), Nordeste de Roraima (municípios de Bonfim, Cantá, Normandia e Uiramutã) e Sudeste de Roraima (municípios de Caroebe, São João da Baliza, São Luis do Anauá e Rorainópolis). O estado conta com um efetivo de 4.732 mil estabelecimentos pecuários criadores de bovinos e com aproximadamente 663.051 bovinos (dadosfornecidos pela Agência de Defesa Agropecuária do Estado de Roraima - ADERR).

\section{Levantamento de dados e diagnóstico sorológico}

A ADERR possui dez Unidades Locais de Sanidade Animal e Vegetal, quatro gerências regionais e uma unidade central. Foram utilizados dados fornecidos pela Gerência Operacional de Defesa Animal provenientes dos condensados estaduais mensais dos informes sobre diagnóstico da brucelose que são obtidos após análise dos relatórios mensais emitidos por médicos veterinários das diferentes microrregiões do Estado, habilitados pelo MAPA para diagnóstico da brucelose bovina. O período do estudo foi de janeiro de 2007 a julho de 2009.

Para o diagnóstico sorológico da infecção por Brucella abortus foi utilizado o teste do antígeno acidificado tamponado (AAT) (BRASIL, 2006), realizado nas salas de diagnóstico dos médicos veterinários habilitados no PNCEBT no Estado de Roraima. Apenas uma pequena parte dos animais reagentes positivos ao AAT foi submetida a teste confirmatório de fixação de complemento, realizado no Instituto Biológico de São Paulo, por solicitação dos produtores, de maneira que, no presente trabalho, o resultado do teste do AAT foi considerado definitivo.

\section{Análise dos dados}

Uma propriedade foi considerada positiva (foco) quando apresentou pelo menos um animal soropositivo. Foram calculadas as frequências de focos e de animais soropositivos por microrregião, bem como por município. Para a confecção do mapa georreferenciado, foi utilizado o programa ArcGIS versão 9.2.

$\mathrm{Na}$ análise de fatores de risco, foram utilizadas 278 propriedades, nas quais foi realizada uma visita com prévio consentimento dos proprietários para aplicação de questionário epidemiológico. $\mathrm{O}$ questionário foi elaborado para obter informações sobre o tipo de exploração e as práticas de manejo empregadas, de forma a permitir a realização do estudo de fatores de risco associados à presença da brucelose. As variáveis analisadas foram: tipo de exploração (ou sistema de produção: carne, leite e misto), tipo de criação (confinado, semiconfinado, extensivo), uso de inseminação artificial, raças predominantes, tamanho do rebanho, presença de outras espécies domésticas, presença de animais silvestres, ocorrência de abortamentos, compra e venda de reprodutores, vacinação contra brucelose, aluguel de pastos, pastos comuns com outras propriedades, presença de áreas alagadiças, piquete de parição e assistência veterinária.

As variáveis foram organizadas em escala crescente de risco e, quando necessário, realizou-se a recategorização delas. A categoria de menor risco foi considerada como base para a comparação com as demais categorias. Inicialmente foi feita uma análise univariada dos dados pelo teste de qui-quadrado ou teste exato de Fisher. Em seguida, as variáveis que apresentaram $\mathrm{p} \leq 0,20$ foram utilizadas na regressão logística, com nível de significância de $5 \%$. O ajuste do modelo final foi verificado com teste de Hosmer e Lemeshow (HOSMER; LEMESHOW, 2000), no qual um $p \geq 0,05$ indica que o modelo está ajustado. Os cálculos foram realizados com o auxílio do programa SPSS version 12.0. 


\section{RESULTADOS E DISCUSSÃO}

Na Tabela 1 estão apresentados os dados censitários da população bovina existente no Estado de Roraima segundo a microrregião (dados fornecidos pela ADERR). No total, no presente trabalho, foram examinadas 387 propriedades, no período de janeiro de 2007 a julho de 2009. Das propriedades examinadas, $106(27,4 \%)$ apresentaram pelo menos um animal soropositivo para brucelose no teste do AAT (Tabela 2). Em relação aos animais, 9.087 fêmeas bovinas com idade $\geq 24$ meses foram submetidas ao diagnóstico de brucelose, das quais 369 $(4,1 \%)$ foram soropositivas (Tabela 3). Na Tabela 3 e na Figura 1 estão apresentadas as frequências de animais soropositivos por município. A frequência de animais soropositivos variou de 0,0 no Município de Normandia a 9,1\% no Município de São Luís do Anauá. Os municípios de Pacaraima e Uiramutã não foram incluídos no trabalho em decorrência deles estarem localizados em áreas contínuas indígenas (Raposa Serra do Sol).

\section{Animais soropositivos (\%)}
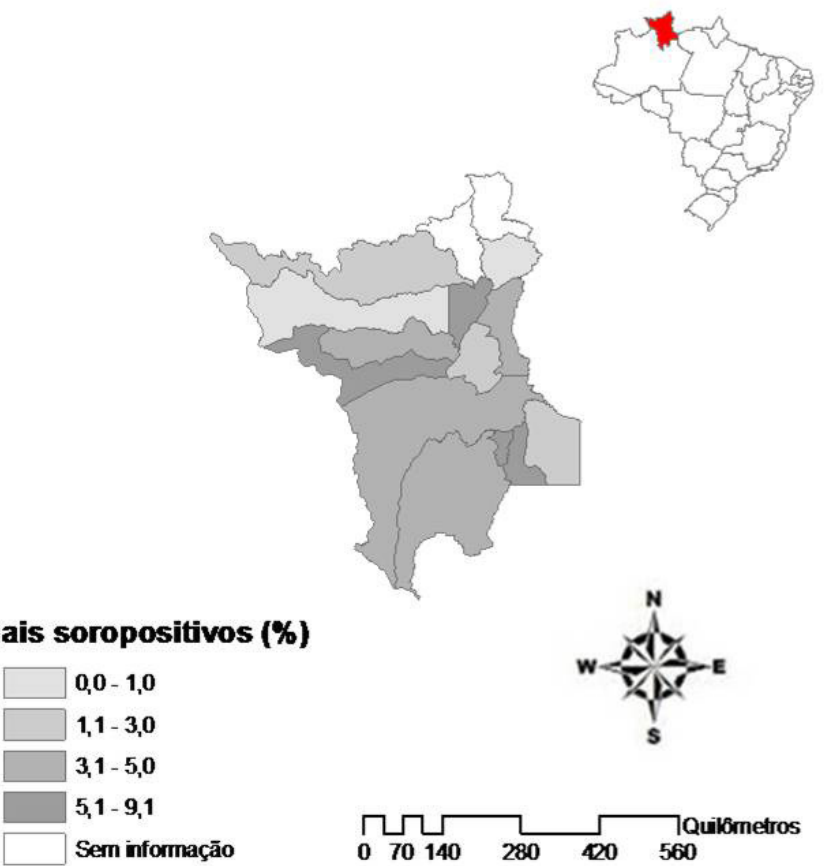

Fig. 1 - Frequência de fêmeas bovinas com idade $\geq 24$ meses soropositivas para brucelose bovina no Estado de Roraima, segundo o município, no período de janeiro de 2007 a julho de 2009.

Tabela 1 - Dados censitários da população bovina existente no Estado de Roraima, segundo as microrregiões.

\begin{tabular}{lccc}
\hline Microrregião & $\begin{array}{c}\text { Total de propriedades } \\
\text { utilizadas }\end{array}$ & $\begin{array}{c}\text { Total de fêmeas bovinas com } \\
\text { idade } \geq 24 \text { meses }\end{array}$ & $\begin{array}{c}\text { Fêmeas bovinas com idade } \geq \\
24 \text { meses utilizadas }\end{array}$ \\
\hline Boa Vista & 137 & 84.531 & 1.180 \\
Caracaraí & 135 & 62.909 & 3.762 \\
Nordeste de Roraima & 23 & 58.327 & 1.886 \\
Sudeste de Roraima & 92 & 57.318 & 2.259 \\
\hline Total & 387 & 263.085 & 9.087 \\
\hline
\end{tabular}

Tabela 2 - Focos de brucelose bovina distribuídos nas quatro microrregiões do Estado de Roraima, no período de janeiro de 2007 a julho de 2009 .

\begin{tabular}{lccc}
\hline Microrregião & Total de propriedades utilizadas & $\begin{array}{c}\text { Propriedades } \\
\text { positivas (focos) }\end{array}$ & Frequência de focos (\%) \\
\hline Boa Vista & 137 & 2 & 19,7 \\
Caracaraí & 135 & 43 & 31,8 \\
Nordeste de Roraima & 23 & 5 & 21,7 \\
Sudeste de Roraima & 92 & 31 & 33,7 \\
\hline Total & 387 & 106 & 27,4 \\
\hline
\end{tabular}


As prevalências de rebanhos com pelo menos um animal soropositivo para $B$. abortus nas Unidades Federativas, em estudos conduzidos como parte do Programa Nacional de Controle e Erradicação da Brucelose e Tuberculose Bovina, variaram de 0,32\% no Estado de Santa Catarina (SIKUSAWA et al., 2009) a 41,5\% no Estado do Mato Grosso do Sul (ChAтE et al., 2009). No presente trabalho, a frequência de focos de brucelose $(27,4 \%)$ pode ser considerada alta, embora não se tenha adotado um planejamento amostral. Essa alta frequência de propriedades positivas pode ser atribuída à proximidade do Estado com a Venezuela e a Guiana, o que pode favorecer a introdução de animais nos rebanhos sem o conhecimento do seu estado sanitário. Por outro lado, a estrutura de produção do Estado,comnúmeroelevadodeanimais, também pode ter contribuído para a elevada frequência de focos. O número médio de cabeças por propriedade foi de 159; neste caso, existe uma tendência de a frequência de focos ser elevada, pois basta um animal positivo para classificar toda o rebanho como positivo, ou seja, uma mesma frequência de animais tende a determinar frequência de focos mais elevada em regiões de propriedades grandes, com número elevado de animais, do que em regiões de propriedades pequenas com pequeno número de animais (CHATE et al., 2009).

A frequência de fêmeas bovinas soropositivas constatada no presente trabalho $(4,1 \%)$ também pode ser considerada alta dada a meta de erradicação da doença, e encontra-se dentro da variação das prevalências obtidas em outros estados brasileiros (0,06\% a 10,2\%) (Alves et al., 2009; AZEVEDO et al., 2009; Chate et al., 2009; DiAs et al., 2009a; DiAs et al., 2009b; GONÇALVES et al., 2009a; GONÇALVES et al., 2009b; Klein-GunNewieK et al., 2009; Marvulo et al., 2009; Negreiros et al., 2009; Ogata et al., 2009; ROCHA et al., 2009; SiKUSAWA et al., 2009; SiLVA et al., 2009; VILLAR et al., 2009).

O combate à brucelose bovina pode ser dividido em quatro fases distintas (PAulin; Ferreira NETO, 2003): (a) rebaixamento da prevalência para valores inferiores a $2 \%$, sendo necessária para isso uma cobertura vacinal de $80 \%$ com a vacina B19; (b) abandono da vacinação e adoção das medidas de diagnóstico e sacrifício sistemáticos dos animais soropositivos; (c) solução de problemas residuais; e (d) adoção de ações de vigilância para que o retorno da infecção seja impedido, ou caso reapareça, seja rapidamente detectado e eliminado. Com base no estudo epidemiológico realizado no Estado de Santa Catarina, no qual a prevalência de focos foi de $0,32 \%$ e a prevalência de animais soropositivos foi de 0,06\% (SIKUSAWA et al., 2009), o MAPA publicou uma portaria em 2004 excluindo a obrigatoriedade de vacinação contra a brucelose bovina nesse estado (BRASIL, 2004).

Tabela 3 - Animais soropositivos para brucelose bovina no Estado de Roraima, conforme o município, no período de janeiro de 2007 a julho de 2009.

\begin{tabular}{|c|c|c|c|}
\hline Municípios & $\begin{array}{c}\text { Fêmeas bovinas com idade } \geq \\
24 \text { meses utilizadas }\end{array}$ & $\begin{array}{c}\text { Fêmeas bovinas com idade } \geq \\
24 \text { meses soropositivas }\end{array}$ & $\begin{array}{l}\text { Frequência de animais } \\
\text { soropositivos (\%) }\end{array}$ \\
\hline Boa Vista & 361 & 27 & 5,3 \\
\hline Alto Alegre & 461 & 4 & 0,9 \\
\hline Amajari & 358 & 5 & 1,4 \\
\hline Cantá & 1.410 & 39 & 2,8 \\
\hline Caracaraí & 507 & 17 & 3,4 \\
\hline Mucajaí & 2.239 & 78 & 3,5 \\
\hline Iracema & 1.016 & 53 & 5,2 \\
\hline Pacaraima & - & - & - \\
\hline Uiramutã & - & - & - \\
\hline Bonfim & 467 & 16 & 3,4 \\
\hline Normandia & 9 & 0 & 0 \\
\hline Rorainópolis & 286 & 9 & 3,2 \\
\hline São Luís do Anauá & 1.325 & 98 & 7,4 \\
\hline São João da Baliza & 198 & 18 & 9,1 \\
\hline Caroebe & 450 & 13 & 2,9 \\
\hline Total & 9.087 & 369 & 4,1 \\
\hline
\end{tabular}


Tabela 4 - Resultados da análise univariada dos possíveis fatores de risco associados à condição de foco de brucelose bovina no Estado de Roraima, no período de janeiro de 2007 a julho de 2009.

\begin{tabular}{lccc}
\hline Variável & Expostos/focos & Expostos/não-focos & $\mathrm{p}$ \\
\hline Exploração de leite & $6 / 142$ & $2 / 136$ & 0,273 \\
Criação semiconfinada & $4 / 142$ & $2 / 136$ & 0,685 \\
Não utilizar inseminação artificial & $138 / 141$ & $132 / 136$ & 0,719 \\
Predominância de raças leiteiras & $1 / 142$ & $0 / 136$ & 0,613 \\
Possuir mais de 51 cabeças & $85 / 142$ & $53 / 136$ & $0,001^{*}$ \\
Presença de ovinos e caprinos & $85 / 142$ & $81 / 136$ & 1,000 \\
Presença de equinos & $103 / 142$ & $91 / 136$ & 0,373 \\
Presença de suínos & $101 / 142$ & 0,447 \\
Presença de aves & $112 / 142$ & $102 / 136$ & 0,532 \\
Ausência de cães & $58 / 142$ & $54 / 136$ & 0,943 \\
Ausência de gatos & $125 / 142$ & $116 / 136$ & 0,621 \\
Presença de animais silvestres & $142 / 142$ & $135 / 136$ & 0,489 \\
Ocorrência de abortamentos & $77 / 142$ & $0,053^{*}$ \\
Realizar testes para brucelose & $103 / 142$ & $87 / 136$ & $0,042^{*}$ \\
Comprar reprodutores & $102 / 142$ & $82 / 136$ & $0,003^{*}$ \\
Vender reprodutores & $100 / 142$ & $43 / 136$ & $0,007^{*}$ \\
Vacinar contra brucelose & $101 / 141$ & $73 / 135$ & $0,013^{*}$ \\
Alugar pasto & $77 / 142$ & $77 / 136$ & $<, 001^{*}$ \\
Pasto comum com outras propriedades & $65 / 142$ & $44 / 136$ & $0,007^{*}$ \\
Presença de áreas alagadiças & $111 / 142$ & $40 / 136$ & $0,006^{*}$ \\
Possuir piquete de parição & $20 / 142$ & $85 / 136$ & $0,022^{*}$ \\
\hline Possuir assistência veterinária & $57 / 141$ & $40 / 135$ & $0,080^{*}$ \\
\hline Variavvis usadas na regresa & & \\
\hline
\end{tabular}

*Variáveis usadas na regressão logística $(\mathrm{p} \leq 0,20)$.

Tabela 5 - Modelo final da regressão logística para os fatores de risco associados à condição de foco de brucelose bovina no Estado de Roraima, no período de janeiro de 2007 a julho de 2009.

\begin{tabular}{lccc}
\hline Fator de risco & Odds ratio & IC 95\% & $\mathrm{p}$ \\
\hline Possuir mais de 51 cabeças & 1,87 & $1,13-3,09$ & 0,015 \\
Alugar pasto & 2,2 & $1,32-3,67$ & 0,003 \\
\hline
\end{tabular}

Teste de Hosmer e Lemeshow: $\chi^{2}=0,326 ; p=0,850$.

No presente trabalho, não foi realizado um planejamento amostral para o Estado, bem como nãoforam estabelecidos critérios de seleção de propriedades e animais, o que pode influenciar na determinação das frequências de focos e animais soropositivos, de modo que se torna incoerente sugerir alguma estratégia de ação. De qualquer maneira, considerando as elevadas frequências de focos e de animais soropositivos, sugere-se que esforços sejam concentrados na intensificação da vacinação de bezerras, com o intuito de que seja alcançada uma prevalência compatível com as ações de teste e sacrifício de animais.

Na análise de fatores de risco associados à condição de foco de brucelose, as variáveis selecionadas na análise univariada foram: possuir mais de 51 cabeças no rebanho $(p=0,001)$, ocorrência de abortamentos $(p=0,053)$, realizar testes para brucelose $(p=0,042)$, comprar reprodutores $(p=0,003)$, vender reprodutores $(p=0,007)$, vacinar contra brucelose $(p$ $=0,013)$, alugar pasto $(\mathrm{p}<0,001)$, pasto comum com outras propriedades $(p=0,007)$, presença de áreas alagadiças $(p=0,006)$, possuir piquete de parição $(\mathrm{p}=0,022)$ e possuir assistência veterinária $(\mathrm{p}=$ $0,080)$ (Tabela 4). No modelo final, possuir mais de 51 cabeças no rebanho (odds ratio $=1,87 ; \mathrm{p}=0,015$ ) e alugar pasto (odds ratio $=2,20 ; \mathrm{p}=0,003$ ) foram apontadas como fatores de risco (Tabelaqq 5). O modelo final apresentou um bom ajuste (Teste de Hosmer e Lemeshow: $\left.\chi^{2}=0,326 ; p=0,850\right) .1$

A associação entre tamanho de rebanhoe presença de brucelose já foi demonstrada (NICOLETTI, 1980; SALMAN; MeYer, 1984; Azevedo et al., 2009; Chate et al., 2009). Em rebanhos grandes, uma vez introduzida a doença, quanto maior o número de bovinos existentes maior será a proporção de animais expostos, de modo que a doença torna-se mais persistente e de difícil controle e erradicação (SALMAn; MEYER, 1984; CRAWFORD et al., 1990). De certa forma, o tamanho do rebanho (número total de bovinos) deve ser encarado como uma variável de confusão em vez de um fator de risco, pois várias práticas podem estar associadas ao tamanho do rebanho e à ocorrência de brucelose bovina, como, por exemplo, a compra de animais, ou seja, em muitos casos, rebanhos grandes são 
mantidos pela compra de animais. CHRIsTIE (1969) observou que o aumento do rebanho resulta em aumento da probabilidade de ocorrência e persistência da infecção, e no aumento da prevalência da doença e da dificuldade de erradicá-la. Assim, número elevado de animais no rebanho significa maior risco de introdução e disseminação da brucelose.

A prática de alugar pastos como fator de risco indica contato indireto entre rebanhos e foi um fato esperado dada a possibilidade de contaminação de pastagens e de água por animais de outras propriedades. O agente, uma vez presente no ambiente, pode permanecer viável por longos períodos, dependendo das condições de umidade, temperatura e sombreamento, aumentando de forma significativa a chance de contato e infecção de um novo indivíduo susceptível (NiCOLETTI, 1980; CRAWFORD et al., 1990; ACHA; SZYFRES, 2001). Uma sugestão para tais propriedades seria o aluguel de pasto apenas para pastejo de animais procedentes de propriedades certificadas como livres ou monitoradas para a brucelose bovina ou de animais soronegativos para a brucelose bovina.

\section{CONCLUSÕES}

Com base nos resultados obtidos no presente trabalho, conclui-se que as frequências de propriedades positivas (focos) $(27,4 \%$ ) e de animais soropositivos $(4,1 \%)$ para brucelose bovina no Estado de Roraima, no período de janeiro de 2007 a julho de 2009, foram elevadas. Dessa maneira, a despeito da ausência de planejamento amostral adequado, sugere-se que esforços sejam concentrados na intensificação da vacinação de bezerras, com o intuito de que seja alcançada uma prevalência compatível com as ações de teste e sacrifício de animais.

\section{AGRADECIMENTOS}

À Agência de Defesa Agropecuária do Estado de Roraima (ADERR) pela concessão dos dados.

\section{REFERÊNCIAS}

ACHA, P.N.; SZYFRES, B. Zoonosis y enfermedades transmisibles comunes al hombre y a los animales: bacterioses e micoses. 3.ed. Washington: OPAS, 2001. 416p.

ALVES, A.J.S.; GONÇALVES, V.S.P.; FIGUEIREDO, V.C.F.; LÔBO, J.R.; BAHIENSE, L.; AMAKU, M.; FERREIRA, F.; FERREIRA NETO, J.S.; DIAS, R.A. Situação epidemiológica da brucelose bovina no Estado da Bahia. Arquivo Brasileiro de Medicina Veterinária e Zootecnia, v.61, p.6-13, 2009. Suplemento 1.
AZEVEDO, S.S.; FERREIRA NETO, J.S.; DIAS, R.A.; FERREIRA, F.; AMAKU, M.; FIGUEIREDO, V.C.F.; LÔBO, J.R.; GONÇALVES, V.S.P.; SOUZA, A.C.; VASCONCELLOS, S.A. Situação epidemiológica da brucelose bovina no Estado do Espírito Santo. Arquivo Brasileiro de Medicina Veterinária e Zootecnia, v.61, p.1926, 2009. Suplemento 1.

BRASIL. Ministério da Agricultura, Pecuária e Abastecimento. Secretaria de Defesa Agropecuária. Departamento de Defesa Animal. Instrução Normativa n.2, de 10 de janeiro de 2001, Programa Nacional de Controle e Erradicação da Brucelose e Tuberculose Animal. Diário Oficial da República Federativa do Brasil, Poder Executivo, Brasília, DF, 16 de janeiro de 2001, Seção 1, p.11-17.

BRASIL. Ministério da Agricultura, Pecuária e Abastecimento. Secretaria de Defesa Agropecuária. Departamento de Defesa Animal. Exclui o Estado de Santa Catarina da obrigatoriedade de vacinação das fêmeas bovinas e bubalinas contra a brucelose. Portaria n.11, de 26 de janeiro de 2004. Diário Oficial da União, Brasília, 29 de janeiro de 2004, Seção 1, p.3.

BRASIL. Ministério da Agricultura, Pecuária e Abastecimento. Programa Nacional de Controle e Erradicação da Brucelose e da Tuberculose Animal (PNCEBT). Manual Técnico. Brasília, DF, 2006. 188p.

CHATE, S.C.; DIAS, R.A.; AMAKU, M.; FERREIRA, F.; MORAES, G.M.; COSTA NETO, A.A.; MONTEIRO, L.A.R.C.; LÔBO, J.R.; FIGUEIREDO, V.C.F.; GONÇALVES, V.S.P.; FERREIRA NETO, J.S. Situação epidemiológica da brucelose bovina no Estado do Mato Grosso do Sul. Arquivo Brasileiro de Medicina Veterinária e Zootecnia, v.61, p.46-55, 2009.Suplemento 1.

CHRISTIE, T.E. Eradication of brucellosis in northern Ireland: Field problems and experiences. Veterinary Record, v.85, p.268-269, 1969.

CRAWFORD, R.P.; HUBER, J.D.; ADAMS, B.S. Epidemiology and surveillance. In: NIELSEN, K.; DUNCAN, J.R. Animal brucellosis. Boca Raton: CRC Press, 1990. p.131-151.

DIAS, J.A.; MÜLLER, E.E.; DIAS, R.A.; FREITAS, J.C.; AMAKU, M.; FERREIRA, F.; SILVA, M.C.P.; LÔBO, J.R.; FIGUEIREDO, V.C.F.; GONÇALVES, V.S.P.; FERREIRA NETO, J.S. Situação epidemiológica da brucelose bovina no Estado do Paraná. Arquivo Brasileiro de Medicina Veterinária e Zootecnia, v.61, p.66-76, 2009a. Suplemento 1.

DIAS, R.A.; GONÇALVES, V.S.P.; FIGUEIREDO, V.C.F.; LÔBO, J.R.; LIMA, Z.M.B.; PAULIN, L.M.S.; GUNNEWIEK, M.F.K.; AMAKU, M.; FERREIRA NETO, J.S.; FERREIRA, F. Situação epidemiológica da brucelose bovina no Estado de São Paulo. Arquivo Brasileiro de Medicina Veterinária e Zootecnia, v.61, p.118-125, 2009b. Suplemento 1. 
GONÇALVES, V.S.P.; RIBEIRO, L.A.; CALDAS, R.A.; FRANCISCO, P.F.C.; DIAS, R.A.; FERREIRA, F.; AMAKU, M.; FERREIRA NETO, J.S.; FIGUEIREDO, V.C.F.; LÔBO, J.R.; BORGES, J.R.J. Situação epidemiológica da brucelose bovina no Distrito Federal. Arquivo Brasileiro de Medicina Veterinária e Zootecnia, v.61, p.1418, 2009a. Suplemento 1.

GONÇALVES, V.S.P.; DELPHINO, M.K.V.C.; DIAS, R.A.; FERREIRA, F.; AMAKU, M.; FERREIRA NETO, J.S.; PORTO, T.B.; ALVES, C.M.; FIGUEIREDO, V.C.F.; LÔBO, J.R. Situação epidemiológica da brucelose bovina no Estado de Minas Gerais. Arquivo Brasileiro de Medicina Veterinária e Zootecnia, v.61, p.35-45, 2009b. Suplemento 1.

HOSMER, D.W.; LEMESHOW, S. Applied logistic regression. New York: John Wiley \& Sons, 2000. 375p.

IBGE - Instituto Brasileiro de Geografia e Estatística. Censo Agropecuário de 2006. 2006. Disponível em: $<$ http://www.ibge.com.br/estadosat/temas. php?sigla=pb\&tema=censoagro $>$. Acesso em: 10 out. 2009.

KLEIN-GUNNEWIEK, M.F.C.; AMAKU, M.; DIAS, R.A.; FERREIRA, F.; GITTI, C.B.; PEREIRA, L.A.; FIGUEIREDO, V.C.F.; LOBO, J.R.; GONÇALVES, V.S.P.; FERREIRA NETO, J.S. Situação epidemiológica da brucelose bovina no Estado do Rio de Janeiro. Arquivo Brasileiro de Medicina Veterinária e Zootecnia, v.61, p.7784, 2009. Suplemento 1.

MARVULO, M.F.V.; FERREIRA, F.; DIAS, R.A.; AMAKU, M.; GROFF, A.C.M.; GONÇALVES, V.S.P.; FIGUEIREDO, V.C.F.; LÔBO, J.R.; FERREIRA NETO, J.S. Situação epidemiológica da brucelose bovina no Estado do Rio Grande do Sul. Arquivo Brasileiro de Medicina Veterinária e Zootecnia, v.61, p.93-102, 2009. Suplemento 1.

MATHIAS, L.A.; MEIRELLES, R.B.; BUCHALA, F.G. Estabilidade do antígeno de célula total de Brucella abortus para uso no diagnóstico sorológico da brucelose bovina pela reação de fixação de complemento. Pesquisa Veterinária Brasileira, v.27, n.1, p.18-22, 2007.

MEGID, J.; RIBEIRO, M.G.; MARCOS, G. JR.; CROCCI, A.J. Avaliação das provas de soroaglutinação rápida, soroaglutinação lenta, antígeno acidificado e 2-mercaptoetanol no diagnóstico da brucelose bovina. Brazilian Journal of Veterinary Research and Animal Science, v.37, n.5, 2000. Disponível em: <http:/ / www. scielo.br/scielo.php?script=sci_arttext\&pid=S1413 -95962000000500009\&lng=pt\&nrm=iso\&tlng=pt $>$. Acesso em: 10 out. 2009.

NEGREIROS, R.L.; DIAS, R.A.; FERREIRA, F.; FERREIRA NETO, J.S.; GONÇALVES, V.S.P.; SILVA, M.C.P.; FIGUEIREDO, V.C.F.; LÔBO, J.R.; FREITAS, J.; AMAKU, M. Situação epidemiológica da brucelose bovina no Estado de Mato Grosso. Arquivo Brasileiro de Medicina Veterinária e Zootecnia, v.61, p.6-65, 2009. Suplemento 1.

NICOLETTI, P. The epidemiology of bovine brucellosis. Advances in Veterinary Science and Comparative Medicine, v.24, p.69-98, 1980.

OGATA, R.A.; GONÇALVES, V.S.P.; FIGUEIREDO, V.C.F.; LÔBO, J.R.; RODRIGUES, A.L.; AMAKU, M.; FERREIRA, F.; FERREIRA NETO, J.S.; DIAS, R.A. Situação epidemiológica da brucelose bovina no Estado do Tocantins. Arquivo Brasileiro de Medicina Veterinária e Zootecnia, v.61, p.126-134, 2009. Suplemento 1.

PAULIN, L.M.; FERREIRA NETO J.S.O Combate à brucelose bovina: situação brasileira Jaboticabal: Funep, 2003. $154 \mathrm{p}$.

POESTER, F.P.; GONÇALVES, V.S.P.; LAGE, A.P. Brucellosis in Brazil. Veterinary Microbiology, v.90, p.55-62, 2002.

ROCHA, W.V.; GONÇALVES, V.S.P.; COELHO, C.G.N.F.L.; BRITO, W.M.E.D.; DIAS, R.A.; DELPHINO, M.K.V.C.; FERREIRA, F.; AMAKU, M.; FERREIRA NETO, J.S.; FIGUEIREDO, V.C.F.; LÔBO, J.R.; BRITO, L.A.B. Situação epidemiológica da brucelose bovina no Estado de Goiás. Arquivo Brasileiro de Medicina Veterinária e Zootecnia, v.61, p.27-34, 2009. Suplemento 1.

SALMAN, M.D.; MEYER, M.E. Epidemiology of bovine brucellosis in the Mexicali Valley, Mexico: literature review of disease-associated factors. American Journal of Veterinary Research, v.45, n.8, p.1561-1566, 1984.

SIKUSAWA, S.; AMAKU, M.; DIAS, R. A.; FERREIRA NETO, J. S.; MARTINS, C.; GONÇALVES, V. S. P.; FIGUEIREDO, V. C. F.; LÔBO, J. R.; FERREIRA, F. Situação epidemiológica da brucelose bovina no Estado de Santa Catarina. Arquivo Brasileiro de Medicina Veterinária e Zootecnia, v.61, p.103-108, 2009. Suplemento 1.

SILVA, V.G.S.O.; DIAS, R.A.; FERREIRA, F.; AMAKU, M.; COSTA, E.L.S.; LÔBO, J.R.; FIGUEIREDO, V.C.F.; GONÇALVES, V.S.P.; FERREIRA NETO, J.S. Situação epidemiológica da brucelose bovina no Estado de Sergipe. Arquivo Brasileiro de Medicina Veterinária e Zootecnia, v.61, p.109-117, 2009. Suplemento 1.

VILLAR, K.S.; AMAKU, M.; DIAS, R.A.; FERREIRA NETO, J.S.; BENITEZ, F.; GONÇALVES, V.S.P.; FIGUEIREDO, V.C.F.; LÔBO, J.R.; FERREIRA, F. Situação epidemiológica da brucelose bovina no Estado de Rondônia. Arquivo Brasileiro de Medicina Veterinária e Zootecnia, v.61, p.85-92, 2009. Suplemento 1.

Recebido em 10/5/11

Aceito em 29/6/12 